\title{
Preparation and Electromagnetic Properties of an Electromagnetic Wave Absorber
}

\author{
Sun Chang ${ }^{1,2,3 *}$, Sun Kangning ${ }^{3}$, Pang Laixue ${ }^{4}$, and Liu Jian ${ }^{2}$ \\ ${ }^{1}$ Co-Innovation Center of Green Building, Shandong Jianzhu University, Jinan 250101, China \\ ${ }^{2}$ School of Material Science and Engineering, Shandong Jianzhu University, Jinan 250101, China \\ ${ }^{3}$ Engineering Ceramics Key Laboratory of Shandong Province, Shandong University, Jinan 250061, China \\ ${ }^{4}$ Research Center for Road Safety, Emergency, Disaster Reduction Technology, Shandong Jiaotong University, Jinan 250357, China
}

(Received 14 December 2015, Received in final form 27 January 2016, Accepted 1 February 2016)

\begin{abstract}
In this study, we report the as-prepared $\mathrm{MgO}$-doped $\mathrm{BaFe}_{12} \mathrm{O}_{19}$, which was prepared by calcination technique and high-energy ball milling process, as an electromagnetic wave absorber. The phase analysis of $\mathrm{BaFe}_{12} \mathrm{O}_{19}$ and the as-prepared $\mathrm{MgO}$-doped $\mathrm{BaFe}_{12} \mathrm{O}_{19}$ was detected utilizing X-ray Diffractometer (XRD). The microstructure was characterized using Scanning Electron Microscope (SEM). By means of the transmission/ reflection coaxial line method, the electromagnetic properties and microwave absorbing properties of the asprepared electromagnetic wave absorber were studied. It is found that the electromagnetic wave absorber has a minimum reflection loss value of $-41 \mathrm{~dB}$ at $4.27 \mathrm{GHz}$ with a matching thickness of $2.6 \mathrm{~mm}$. The experiment results revealed that the as-prepared electromagnetic wave absorber could find potential applications in many military as well as commercial industries.
\end{abstract}

Keywords : $\mathrm{MgO}$-doped $\mathrm{BaFe}_{12} \mathrm{O}_{19}$, microwave absorbing properties, microwave absorber

\section{Introduction}

With the development of communication devices which use the electromagnetic waves of $\mathrm{GHz}$ range, the problems of electromagnetic interference (EMI) have become increasingly serious $[1,2]$. Adopting microwave absorbing materials to convert the electromagnetic waves energy into heat is one of the most effective methods for eliminating the above-mentioned problems. Moreover, radar absorbing materials (RAM) play a significant role in military stealth technologies [3, 4]. To satisfy the needs of military and commercial applications, therefore, the microwave absorbing materials have been extensively investigated [5-8]. Barium ferrite $\left(\mathrm{BaFe}_{12} \mathrm{O}_{19}\right)$, which possesses excellent physical properties has long been used as an important microwave absorber [9-11]. In order to be accustomed to specific applications, a lot of work has been done to optimize electromagnetic parameters of barium ferrites, such as substitutions for $\mathrm{Fe}^{3+}$ with other cations, shape, modified composition, and so on [12-16]. In particular, some oxides, which include $\mathrm{Ho}_{2} \mathrm{O}_{3}$ [17],

(C)The Korean Magnetics Society. All rights reserved.

*Corresponding author: Tel: +8613589035333

Fax:+8653186367286, e-mail: sunchang@sdu.edu.cn
$\mathrm{TiO}_{2}$ [18], $\mathrm{SnO}_{2}$ [19], $\mathrm{Ga}_{2} \mathrm{O}_{3}$ [20], $\mathrm{Sm}_{2} \mathrm{O}_{3}$ [21], $\mathrm{Co}_{3} \mathrm{O}_{4}$ [22], etc., were doped into $\mathrm{BaFe}_{12} \mathrm{O}_{19}$ to regulate and improve its physical properties. Serving as a conventional additive, magnesium oxide ( $\mathrm{MgO})$ has been successfully added to some ceramics such as BSTO [23], $\mathrm{Bi}_{0.5} \mathrm{Na}_{0.5} \mathrm{TiO}_{3}$ [24], $\mathrm{ZrO}_{2}$ [25], AlN [26], $\mathrm{Ba}_{3}\left(\mathrm{Co}_{0.4} \mathrm{Zn}_{0.6}\right)_{2} \mathrm{Fe}_{23.6} \mathrm{O}_{41}$ [27], $\mathrm{LiNbO}_{3}$ [28], etc. $\mathrm{MgO}$ as sintering aids is believed to be able to improve densification and avoid the tendency of discontinuous grain growth during the sintering process. However, very few reports concerned regarding MgOdoped $\mathrm{BaFe}_{12} \mathrm{O}_{19}$ to the best of the authors knowledge. The purpose of this paper is, therefore, to prepare MgOdoped $\mathrm{BaFe}_{12} \mathrm{O}_{19}$ by calcination technique and highenergy ball milling process. The microwave absorption properties, phase composition, topography of the asprepared $\mathrm{MgO}$-doped $\mathrm{BaFe}_{12} \mathrm{O}_{19}$ have been investigated.

\section{Materials and Methods}

All regents used were of analytical purity and used without further purification in the present work. The $\mathrm{BaFe}_{12} \mathrm{O}_{19}$ powder was synthesized by sol-gel method with brief details as the following. The as-prepared $\mathrm{BaFe}_{12} \mathrm{O}_{19}$ powder was mixed with $1 \mathrm{wt} \% \mathrm{MgO}$ and was well dispersed through high energy ball-milling in a QM- 
BP planetary ball mill for $5 \mathrm{~h}$ at a rotation speed of about $400 \mathrm{rpm}$. The ball-to-composite mass ratio was 6:1. The mixture of $\mathrm{BaFe}_{12} \mathrm{O}_{19}$ and $\mathrm{MgO}$ particle was subsequently cold pressed into pellets under $300 \mathrm{MPa}$ pressure, and was subsequently calcined at $1030^{\circ} \mathrm{C}$ in an electric resistance furnace for $12 \mathrm{~h}$ to form a dense sintered body of $\mathrm{MgO}$-doped $\mathrm{BaFe}_{12} \mathrm{O}_{19}$. Then, the fine $\mathrm{MgO}$-doped $\mathrm{BaFe}_{12} \mathrm{O}_{19}$ powders with an average grain size of $200 \mathrm{~nm}$ were prepared after the as-prepared pellets were ballmilled in a QM-BP planetary ball mill. Finally, powdery electromagnetic wave absorber was obtained.

Phase composition of the as-prepared electromagnetic wave absorber was identified by means of an X-ray diffractometer (RIGAKUD/Max-A) using $\mathrm{Cu} \mathrm{K} \alpha$ radiation $(\lambda=1.5405)$ at a $2 \theta$ range of $10-70^{\circ}$. The morphology of the as-prepared electromagnetic wave absorber was characterized via scanning electron microscope (SEM, JSM-6700F). In order to study the electromagnetic parameters and microwave absorbing properties, the asprepared electromagnetic wave absorber was molded into toroidally-shaped samples (with an inner diameter of 3.04 $\mathrm{mm}$, outer diameter of $7 \mathrm{~mm}$, and thickness of about 3 $\mathrm{mm}$ ), mixed with 30 mass $\%$ paraffin wax. The toroidallyshaped samples were inserted in a standard coaxial sample holder, and reflection coefficient ( $S_{11}$ parameter) and transmission coefficient $\left(S_{21}\right.$ parameter) were measured by an AV3618 network analyzer in the range from 0.5 to $6 \mathrm{GHz}$. The complex permittivity $\left(\varepsilon=\varepsilon^{\prime}-j \varepsilon^{\prime \prime}\right)$, complex permeability $\left(\mu=\mu^{\prime}-j \mu^{\prime \prime}\right)$ and reflection loss $(R L)$ of the as-prepared electromagnetic wave absorber was calculated from $S_{11}$ and $S_{21}$ parameters by the transmission/reflection coaxial line method [29].

\section{Results and Discussion}

The typical XRD patterns of (a) $\mathrm{BaFe}_{12} \mathrm{O}_{19}$ prepared with sol-gel process and (b) the as-prepared electromagnetic wave absorber are shown in Fig. 1. From Figure 1(a), it was found that $\mathrm{BaFe}_{12} \mathrm{O}_{19}$ prepared by sol-gel method comprised of pure phase. Figure 1(b) indicates that all peaks could be indexed to the standard patterns as reported by the Joint Committee on Composite Diffraction Standards (JCPDS) for hexagonal $\mathrm{BaFe}_{12} \mathrm{O}_{19}$ (PDF\#43-0002). The characteristic peaks of $\mathrm{MgO}$ are not detected in the patterns. The results reveal that $\mathrm{MgO}$ dissolved in $\mathrm{BaFe}_{12} \mathrm{O}_{19}$ lattice. In addition, compared with pattern (a) in Figure 1, the peaks of the as-prepared electromagnetic wave absorber moved to higher diffraction angles. It is believed that the right shift rose from dissolving $\mathrm{MgO}$ into the lattice of $\mathrm{BaFe}_{12} \mathrm{O}_{19}$ phase [30]. Fig. 2 shows the SEM image of the as-prepared electro-

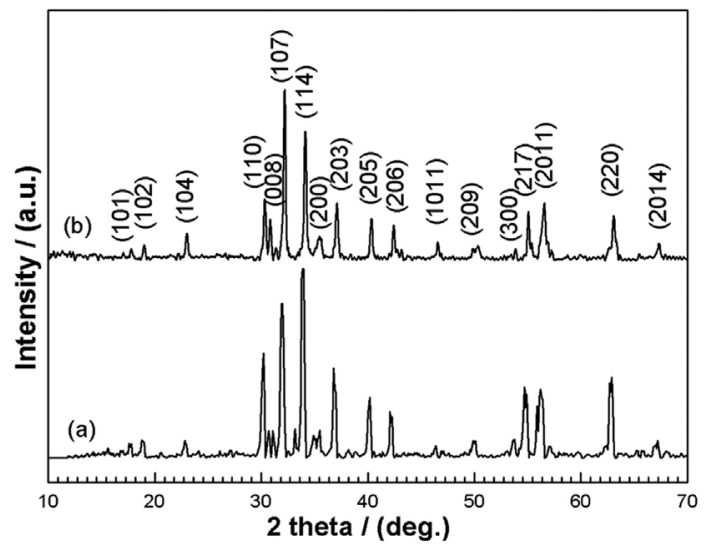

Fig. 1. X-ray diffraction patterns of $\mathrm{BaFe}_{12} \mathrm{O}_{19}$ (a) and as-prepared electromagnetic wave absorber (b).

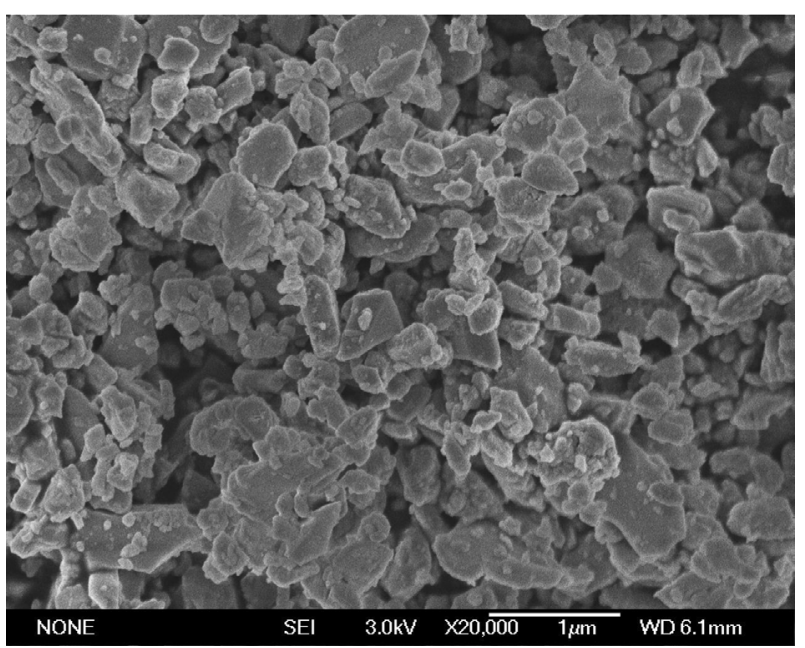

Fig. 2. The SEM micrograph of the as-prepared electromagnetic wave absorber.

magnetic wave absorber. It displays flaky microstructure which is believed to be the best structure for microwave absorber. The particle size of the as-prepared electromagnetic wave absorber was about $200 \mathrm{~nm}$.

Figure 3 shows the frequency dependence of the complex permeability $\left(\varepsilon^{\prime}\right.$ and $\left.\varepsilon^{\prime}\right)$ the the range varied from 0.5 to $6 \mathrm{GHz}$ for the as-prepared electromagnetic wave absorber. It shows that the real part of permittivity declined from 12.3 to 1.4 with the increases of the frequency. Except for a minimum value at $1.9 \mathrm{GHz}$, the $\varepsilon$ " values were almost constant within 0.5 to $6 \mathrm{GHz}$. Fig. 4 shows the frequency dependence of complex permeability $\left(\mu^{\prime}\right.$ and $\mu^{\prime}$ ) of the as-prepared electromagnetic wave absorber. From the pattern (shown in Fig. 4), we can see that the imagine part of permeability rose with the increasing frequency and presents a peak at $5.0 \mathrm{GHz}$, then decreases. However, the real part of complex permeability has an 


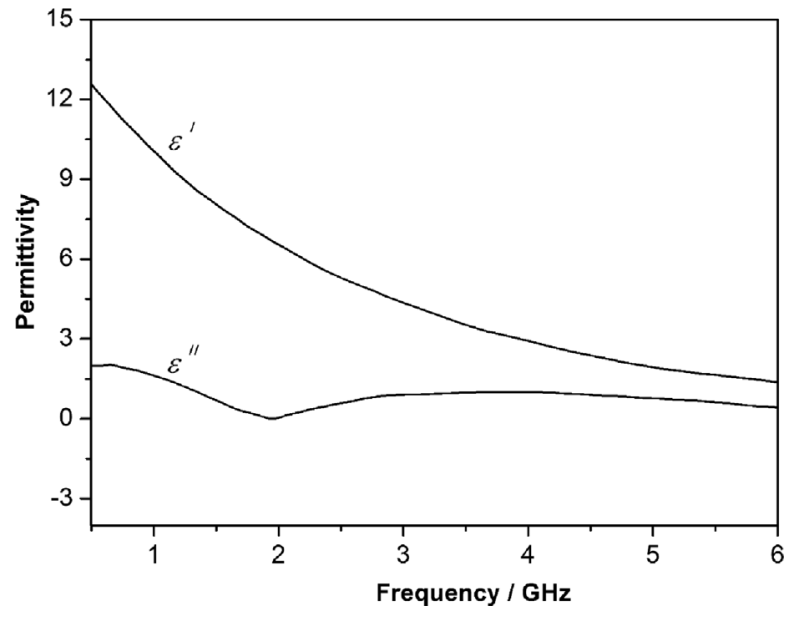

Fig. 3. The permittivity spectra of the as-prepared electromagnetic wave absorber.

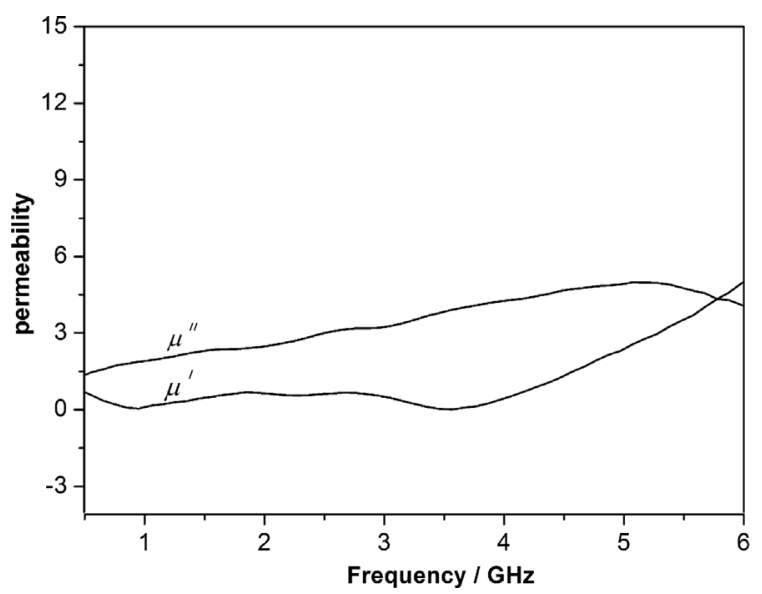

Fig. 4. The permeability spectra of the as-prepared electromagnetic wave absorber.

increasing trend at the range from $3.6 \mathrm{GHz}$ to $6.0 \mathrm{GHz}$.

According to the transmission line theory, the normalized input impedance $\left(Z_{i n}\right)$ at the microwave absorber surface is given by formulas (1) for a microwave absorbing layer that is backed metal plate [31].

$$
Z_{\text {in }}=Z_{0} \sqrt{\mu / \varepsilon} \tanh \{j(2 \pi f d / c) \sqrt{\mu \varepsilon}\}
$$

Where $\mu$ and $\varepsilon$ are the complex permeability $\left(\mu=\mu^{\prime}-\right.$ $\left.j \mu^{\prime \prime}\right)$ and the complex permittivity $\left(\varepsilon=\varepsilon^{\prime}-j \varepsilon^{\prime \prime}\right), f$ is the frequency, $Z_{0}$ is the impedance of air, $c$ is the velocity of electromagnetic waves in free space, and $d$ is the thickness of the absorber. The reflection loss $(R L)$ can be expressed as formula (2)[16].

$$
R L=20 \log \left|\left(Z_{\text {in }}-Z_{o}\right) /\left(Z_{\text {in }}+Z_{o}\right)\right|
$$

From formula (1) and (2), the reflection loss for the asprepared electromagnetic wave absorber can be calcu-

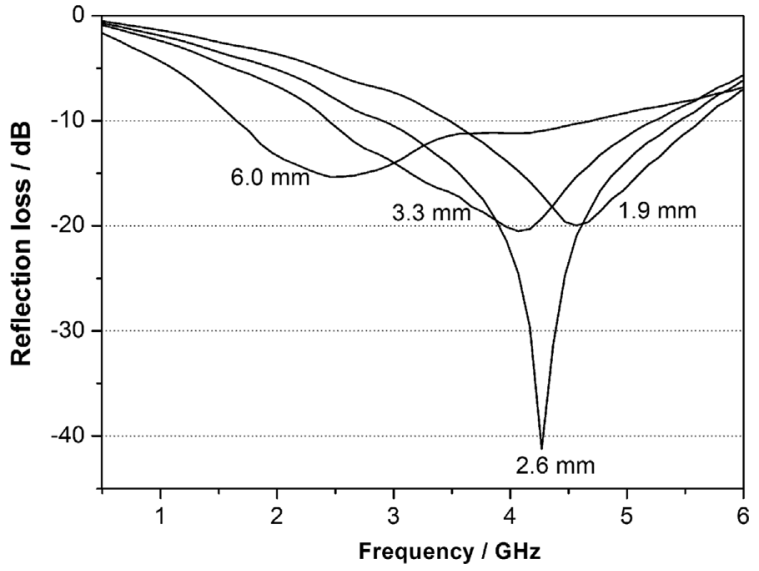

Fig. 5. The calculated reflection loss of the as-prepared electromagnetic wave absorber at different matching thickness.

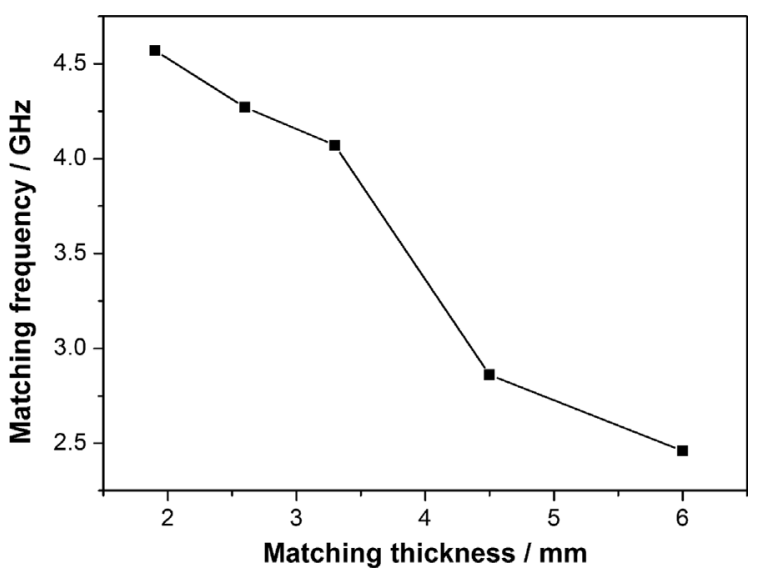

Fig. 6. Relation between matching frequency and matching thickness for the as-prepared electromagnetic wave absorber.

lated. Obviously, the reflection loss is negative value. Furthermore, the lower the reflection loss is, the stronger the electromagnetic wave absorbing ability is. Figure 5 shows the frequency dependence of the calculated reflection loss of the as-prepared electromagnetic wave absorber at the thickness of 1.9, 2.6, 3.3, and $6 \mathrm{~mm}$, respectively. It is found that the minimum value of reflection loss has very close relationship with the material's thickness. The bandwidth with the reflection loss less than $-10 \mathrm{~dB}$ reaches more than $3 \mathrm{GHz}$ with a matching thickness of $6.0 \mathrm{~mm}$. typically, the reflection loss was less than $-20 \mathrm{~dB}$ at the range from 4.07 to $4.57 \mathrm{GHz}$, and the minimum reflection loss value reached $-41 \mathrm{~dB}$ at $4.27 \mathrm{GHz}$ with a matching thickness of $2.6 \mathrm{~mm}$. It can be seen that MgOdoped $\mathrm{BaFe}_{12} \mathrm{O}_{19}$ shows more attractive microwave absorbing properties than the undoped $\mathrm{BaFe}_{12} \mathrm{O}_{19}$ in the range of 0.5 to $6 \mathrm{GHz}$ [32]. Evidently $\mathrm{MgO}$-doped $\mathrm{BaFe}_{12} \mathrm{O}_{19}$ have much more effective microwave absorbing effects. Figure 6 shows the relationship of the matching thickness 
and matching frequencies for minimum reflection loss. It is very obvious that the matching frequency linearly reduces with the increase of the matching thickness.

\section{Conclusions}

The as-prepared electromagnetic wave absorber was successfully synthesized from $\mathrm{BaFe}_{12} \mathrm{O}_{19}$ and $\mathrm{MgO}$ powder by calcination process combined with high-energy ballmill. The complex permittivity and permeability spectra and microwave absorbing properties of the as-prepared electromagnetic wave absorber were investigated in the frequency range $0.5-6 \mathrm{GHz}$. It is found that this composite has a minimum reflection loss value of $-41 \mathrm{~dB}$ at $4.27 \mathrm{GHz}$ with a matching thickness of $2.6 \mathrm{~mm}$. The bandwidth with the reflection loss less than $-10 \mathrm{~dB}$ reaches more than $3 \mathrm{GHz}$ with a matching thickness of $6.0 \mathrm{~mm}$. The results suggest that the as-prepared electromagnetic wave absorber can be used as a good microwave absorption candidate in the range of $0.5-6 \mathrm{GHz}$.

\section{Acknowledgements}

The current work received support from the Scientific Research Fund for Doctorates of Shandong Jianzhu University (XNBS1258), China Postdoctoral Science Foundation (No. 200804401138), Shandong Province Postdoctoral Innovation Foundation (No. 200902030), and Applied Basic Research Planning of Ministry of China (2013319817050).

\section{References}

[1] S. S. Kim and I. K. Choi, J. Magn. 2, 25 (1997).

[2] B. Kim, H. Lee, S. Park, and H. Kim, Thin Solid Films 519, 3492 (2011).

[3] X. Liu, Y. Wu, and Z. Zhang, Physica B: Condensed Matter 405, 4393 (2010).

[4] Z. Zhang, R. Fan, Z. Shi, K. Yan, Z. Zhang, X. Wang, and S. Dou, Rsc. Adv. 3, 26110 (2013).

[5] K. Sakai, N. Asano, Y. Wada, and S. Yoshikado, J. Eur. Ceram Soc. 30, 347 (2010).

[6] M. Itoh, J. R. Liu, T. Horikawa, and K. I. Machida, J. Alloys Compd. 408, 1400 (2006).

[7] K. Yan, R. Fan, M. Chen, K. Sun, L. Yin, H. Li, S. Pan, and M. Yu, J. Alloys Compd. 628, 429 (2015).

[8] M. Chen, R. Fan, M. Gao, S. Pan, M. Yu, and Z. Zhang, J. Magn. Magn. Mater 381, 105 (2015).
[9] S. Y. An, S. W. Lee, I. B. Shim, S. R. Yun, and C. S. Kim, J. Magn. 6, 23 (2001).

[10] V. G. Harris, A. Geiler, Y. Chen, S. D. Yoon, M. Wu, A. Yang, Z. Chen, P. He, P. V. Parimi, and X. Zuo, J. Magn. Magn. Mater 321, 2035 (2009).

[11] M. Meshram, N. K. Agrawal, B. Sinha, and P. Misra, J. Magn. Magn. Mater 271, 207 (2004).

[12] G. Mu, N. Chen, X. Pan, H. Shen, and M. Gu, Mater Lett. 62, 840 (2008).

[13] X. Tang and Y. Yang, Appl. Surf. Sci. 255, 9381 (2009).

[14] J. Qiu, H. Shen, and M. Gu, Powder Technol. 154, 116 (2005).

[15] S. Gairola, V. Verma, A. Singh, L. Purohit, and R. Kotnala, Solid State Commun. 150, 147 (2010).

[16] Y. Feng, T. Qiu, and C. Shen, J. Magn. Magn. Mater 318, 8 (2007).

[17] G. M. Rai, M. Iqbal, and K. Kubra, J. Alloys Compd. 495, 229 (2010).

[18] J. Qiu, L. Lan, H. Zhang, and M. Gu, J. Alloys Compd. 453, 261 (2008).

[19] A. González-Angeles, G. Mendoza-Suárez, A. Grusková, R. Dosoudil, and R. Ortega-Zempoalteca, Mater Lett. 58, 2906 (2004).

[20] I. Bsoul and S. Mahmood, J. Alloys Compd. 489, 110 (2010).

[21] W. Lixi, H. Qiang, M. Lei, and Z. Qitu, J. Rare Earth 25, 216 (2007).

[22] K. K. Mallick, P. Shepherd, and R. J. Green, J. Magn. Magn. Mater 312, 418 (2007).

[23] L. Sengupta, E. Ngo, S. Stowell, M. O'day, and R. Lancto, U.S. Patent 5,427,988 (1995).

[24] C. Chou, J. Chen, R. Yang, and S. Chou, Powder Technol. 202, 39 (2010).

[25] J. Brito-Chaparro, A. Reyes-Rojas, M. Bocanegra-Bernal, A. Aguilar-Elguezabal, and J. Echeberria, Mater Chem. Phys. 106, 45 (2007).

[26] S. Kume, M. Yasuoka, N. Omura, and K. Watari, J. Eur. Ceram. Soc. 25, 2791 (2005).

[27] L. Jia, H. Zhang, J. Luo, Y. Liu, and Q. Wen, J. Magn. Magn. Mater 322, 1934 (2010).

[28] P. Sen, P. Sen, R. Bhatt, S. Kar, V. Shukla, and K. Bartwal, Solid State Commun. 129, 747 (2004).

[29] S. Chang, S. Kangning, and C. Pengfei, J. Magn. Magn. Mater 324, 802 (2012).

[30] Y. Naito and K. Suetake, Microwave Theory and Techniques, IEEE Transactions on 19, 65 (1971).

[31] C. Tsay, H. Cheng, Y. Tung, W. Tuan, and C. Lin, Thin Solid Films 517, 1032 (2008).

[32] H. Yang, T. Ye, Y. Lin, and M. Liu, Synthetic. Met. 210, Part B, 245 (2015). 